\section{THORACIC ACTINOMYCOSIS}

BY

EWART M. JEPSON, M.B., M.R.C.P. Medical Registrar, Westminster Hospital

F. CLIFFORD ROSE, M.B., M.R.C.P. Lately Medical Registrar, Westminster Hospital

\section{AND}

RICHARD D. TONKIN, M.D., F.R.C.P. Assistant Physician, Westminster Hospital

Since Zachary Cope (1938) wrote his classic monograph on actinomycosis, considerable advances in the understanding of the pathogenesis of this disease have been made. We wish to present brief reports of two cases of thoracic actinomycosis illustrative of these advances, both of which were initially entirely unsuspected. Pleuritic pain was the presenting symptom in one and a "rheumatism" of the shoulders in the other. Weight loss and polymorphonuclear leucocytosis were common to both.

Actinomycotic infection is not a rare condition, nor is its incidence confined to farmers or horse dealers. Favourable response to antibiotics is another cogent reason for considering it high on the list of possibilities in the differential diagnosis of any obscure infective condition. When overlooked it can give rise to distressing and protracted disability, often with an eventual unhappy outcome, but if diagnosed early and treated correctly there is every justification for optimistic prognosis.

\section{Case 1}

A 47-year-old car salesman was admitted to Westminster Hospital on February 5, 1948, with a four-weeks history of cough, fever, and pleuritic pain.

Chest radiograph showed collapse and consolidation of the right lower lobe, with an overlying encysted effusion (Fig. 1). The erythrocyte sedimentation rate (E.S.R.) was $49 \mathrm{~mm}$. in first hour (Wintrobe) ; Hb 88\% (13 g./100 ml.);

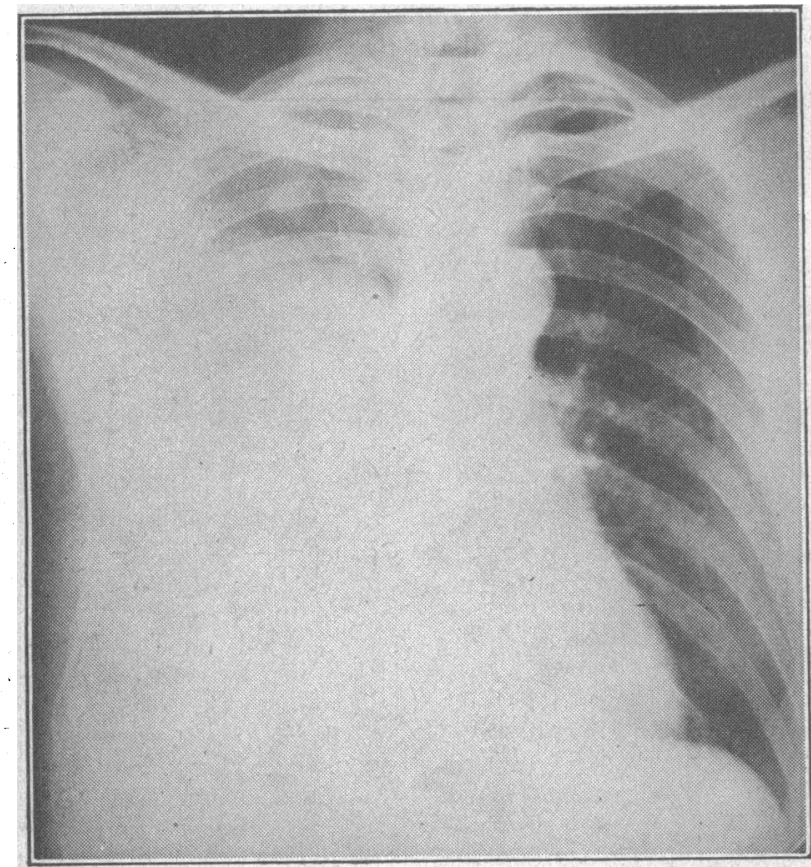

Fig. 1.-Case 1. P.A. chest radiograph February 6, 1948. leucocytes, 12,000 per c.mm. (87\% polymorphs). Tubercle bacilli. were not found in the sputum by microscopy or culture. Three successive pleural taps produced yellow turbid fluid which contained polymorphs but was sterile on aerobic culture. Blood cultures (aerobic and anaerobic) were negative. Two weeks after his admission clubbing of the fingers was observed. Sulphadimidine and penicillin were given but discontinued after one week, having failed to influence the pyrexia. This latter subsided spontaneously over the next month, and the size of the pleural effusion diminished concurrently. The patient was discharged after 10 weeks - pending admission to a sanatorium - with a small residual effusion and a provisional diagnosis of pulmonary tuberculosis. When reviewed as an out-patient three months later he had improved and had gained nearly a stone $(6.4 \mathrm{~kg}$.) in weight, but his fingers were still clubbed and residual signs at the right base persisted.

He was not seen again until seven years later (1955), during which time he had been fully active. His new complaint, of four months' duration only, was of lassitude and loss of over a stone $(6.4 \mathrm{~kg}$.) in weight. He was thin and obviously ill, but afebrile. Gross clubbing of the fingers was present together with physical signs of a right pleural effusion. Chest radiograph revealed a curious and, we think, characteristic type of osteitis of the posterior portions of the fourth to sixth ribs (Fig. 2) in addition to an effusion. E.S.R. $50 \mathrm{~mm}$. in first hour (Wintrobe); leucocytes, 12,000 (90\% polymorphs). Paracentesis yielded thick yellow pus, containing many polymorphs and Grampositive branching rods. After seven days' anaerobic incubation Actinomyces israeli was cultured, and the same organism was recovered from the sputum.

Heavy albuminuria and a urea clearance only $56 \%$ of normal indicated renal damage and a congo-red absorption of $70 \%$ within 30 minutes suggested amyloidosis. This possibility was supported by the finding of splenomegaly.

Six mega units of penicillin was given daily, and continued for three weeks, concurrently with two four-day courses of intramuscular streptomycin, $1 \mathrm{~g}$. a day. At the end of this time rib resection was performed under local analgesia and a thick-walled empyema drained. On the following day the patient felt extremely weak and his temperature fell

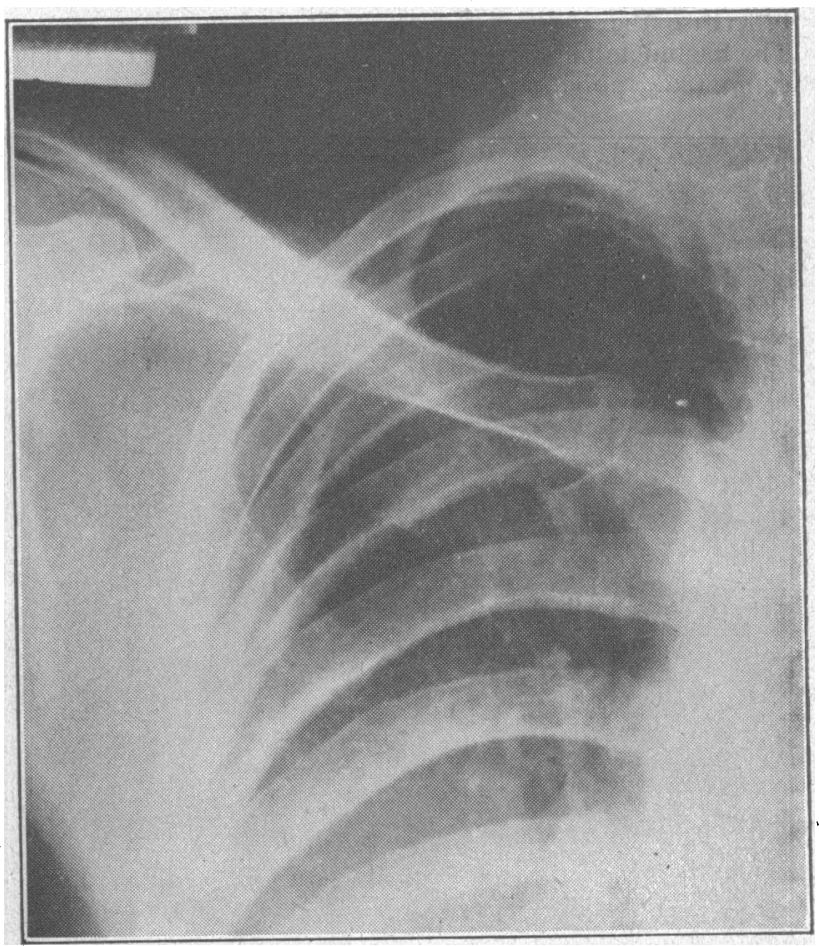

Fig. 2.-Case 1. Enlargement of chest radiograph August 29, 1955, to show right upper ribs. 
dramatically from normal to $95^{\circ} \mathrm{F} .\left(35^{\circ} \mathrm{C}\right.$. $)$ at which level it remained for five days. Two days later a sudden clinical deterioration was accompanied by a fall in blood pressure to a level of $70 / 50 \mathrm{~mm}$. Hg., and the serum electrolytes confirmed the clinical impression of adrenal failure (sodium, $125 \mathrm{mEq} / 1$. ; potassium, $5.9 \mathrm{mEq} / \mathrm{l}$; ; chlorides, $88 \mathrm{mEq} / 1$; blood urea, $114 \mathrm{mg} . / 100 \mathrm{ml}$.). Intravenous infusion containing noradrenaline and $200 \mathrm{mg}$. of hydrocortisone, supplemented intramuscularly by $200 \mathrm{mg}$. of cortisone and $20 \mathrm{mg}$. of deoxycortone acetate, failed to stem his deterioration, and he died the following day.

Post-mortem Examination. - There was a chronic empyema in the right chest, measuring approximately $3 \frac{1}{2}$ in. $(9 \mathrm{~cm}$.) in diameter. Histological examination of the lung revealed non-specific chronic inflammation only. The liver and spleen were moderately enlarged, and showed amyloid reaction, as did the pancreas, adrenals, and kidneys. On histological examination of the adrenal glands the cortex was found to be almost completely replaced by amyloid substance.

Comment.-The case was almost certainly initially suffering from actinomycotic infection, and the later finding of extensive amyloidosis was presumably consequent upon its long-standing presence. In the final stages, failure to appreciate the significance of hypothermia as indicative of adrenal insufficiency delayed substitution therapy, which otherwise might well have altered the outcome. One interesting feature was the characteristic type of rib osteitis, giving an almost diagnostic radiological appearance.

\section{Case 2}

A man aged 49 first presented in the out-patient department of Westminster Hospital in November, 1956. He had experienced a dull interscapular pain for three months which extended into the upper arms and eventually forced him to discontinue work. He admitted to a slight unproductive cough, some nocturnal dyspnoea and sweating, also to a weight loss of 2 stone $(12.7 \mathrm{~kg}$.). On examination he was obviously ill, febrile $-102^{\circ}$ F. $\left(38.9^{\circ} \mathrm{C}\right.$.) -and severely anaemic. There was a subcutaneous, fluctuant, but painless swelling 4 in. (19 cm.) in diameter over the upper dorsal spine, and the overlying skin was not inflamed. Bronchial breathing was audible in the left upper zone anteriorly, but otherwise the chest was free of abnormal physical signs. The haemoglobin was $58 \%$ (8.5 g./100 ml.) ; W.B.C. 18,000/ c.mm. (86\% polymorphs), and E.S.R. of $65 \mathrm{~mm}$. in first hour

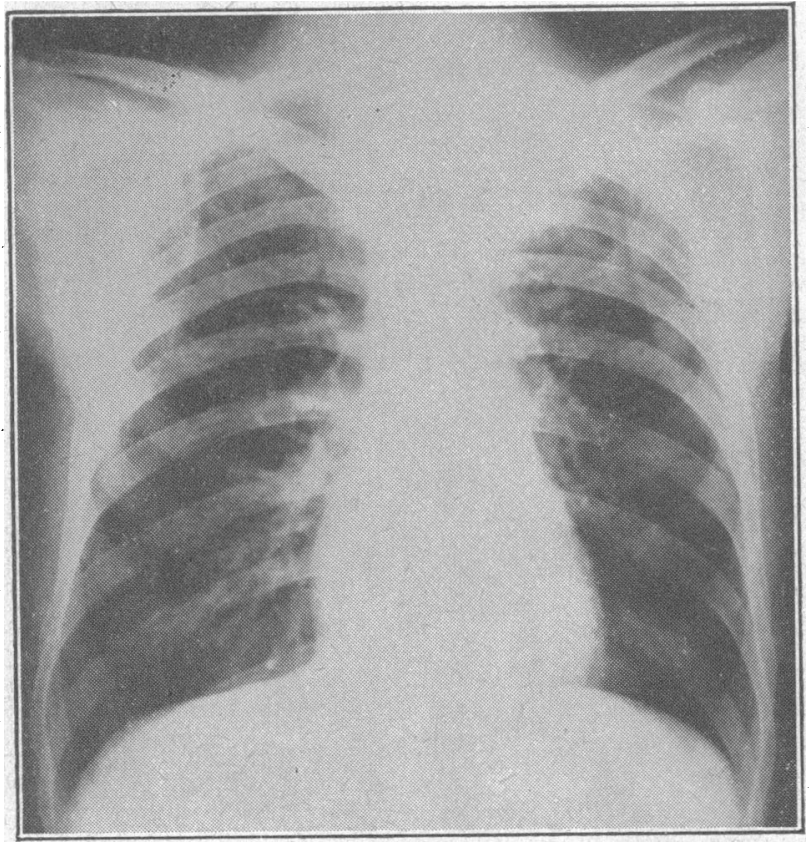

Fig. 3.-Case 2. P.A. chest radiograph November 23, 1956.
(Wintrobe). The chest radiograph showed a homogeneous opacity at the left apex, the rib shadows being normal (Fig. 3). Radiography of the dorsal spine revealed a loss of definition and rarefaction of the bodies of D 1-4 vertebrae (Fig. 4). The abscess was aspirated and $20 \mathrm{ml}$. of brown

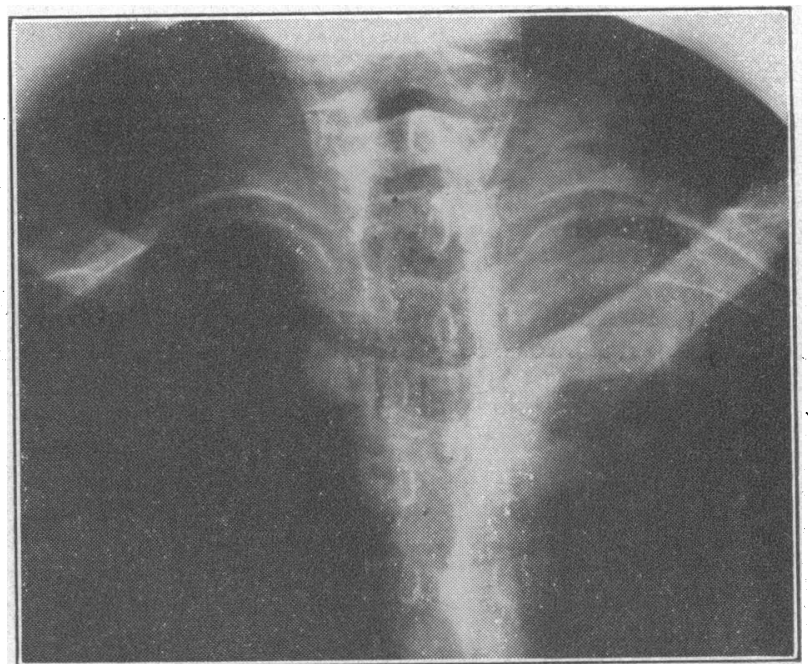

Fig. 4.-Case 2. Radiograph of upper dorsal spine December 5, 1956.

blood-stained pus containing "sulphur granules" withdrawn. Microscopy showed many neutrophils, Grampositive branching filaments, and a moderate number of Gram-negative bacilli. Culture yielded a growth of $A$. israeli and Actinobacillus actinomycetem comitans. Neither of these organisms, nor any tubercle bacilli, were isolated from the sputum.

Six mega units of penicillin was given intramuscularly daily for seven weeks, together with four courses of intramuscular streptomycin, $1 \mathrm{~g}$. a day for four days at fortnightly intervals. Aspiration of the abscess was repeated frequently, and 1 mega unit of penicillin was instilled on each occasion. Fourteen days after treatiment the pus became sterile, and after 28 days no organisms could be demonstrated in the smear. Steady clinical improvement was evidenced by a return of appetite and gain of $13 \mathrm{lb}$. $(5.9 \mathrm{~kg}$.) in weight. The initially high fever fell rapidly but did not return to normal for two weeks. The chest radio-

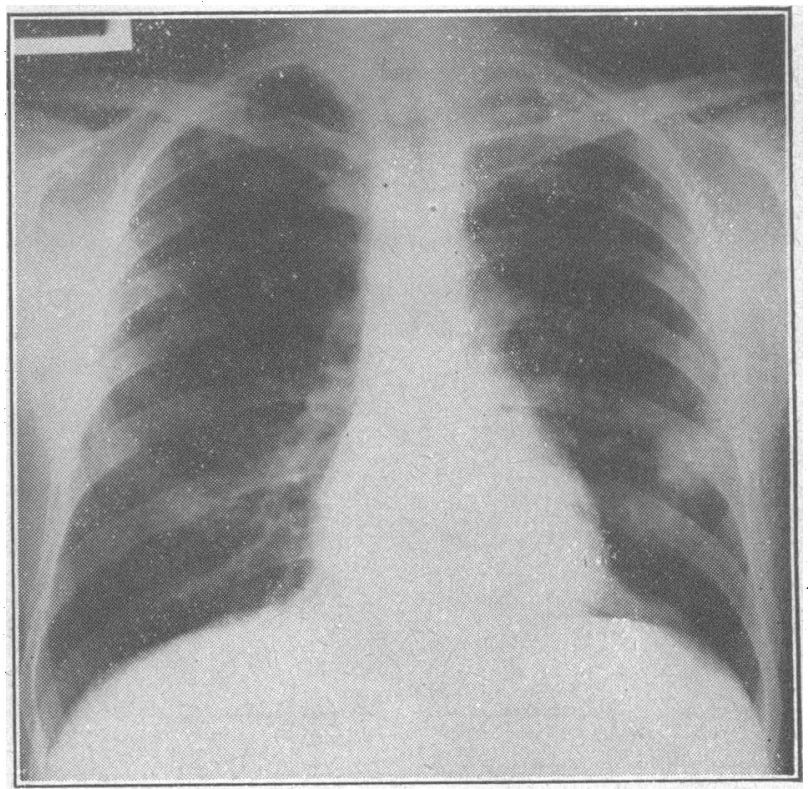

Fic. 5.-Case 2. P.A. chest radiograph April 23, 1957. 
graph showed little change but the appearances of the upper dorsal spine were much improved. He was discharged to convalescence eight weeks after admission.

Four weeks later, although symptom-free, the chest radiograph showed a new opacity in the right second interspace and the left mid-zone (Fig. 5). There was neither pyrexia nor any abnormal physical signs. E.S.R. was $21 \mathrm{~mm}$. in first hour (Wintrobe). Treatment with intramuscular penicillin and streptomycin was given as before for six weeks. The chest radiograph taken at the end of this course showed clearing of both lung fields. He was then given oral phenoxymethylpenicillin, $120 \mathrm{mg}$., three times a day, on which dose he continued.

Comment.-The infection in this instance was either pulmonary or mediastinal, involving the upper dorsal vertebrae and presenting as a cold abscess over the back of the neck. Bilateral intrapulmonary recrudescence following within four weeks of cessation of antibiotic therapy emphasized the necessity for a prolonged follow-up period.

\section{Discussion}

Types of Thoracic Actinomycosis.-These have been classified by Cope (1938) into bronchial, parenchymal, and mediastinal. The first type is rare, with slight symptoms, bronchitis, and actinomyces in the sputum. Parenchymal disease begins generally in the lower lobes with eventual involvement of the remaining lung. Fascial planes are ignored, but lymph nodes and lymphatics are seldom invaded. The pleura shows little resistance, with early effusion, becoming purulent and progressing to empyema formation (Barker, 1954) as in our Case 1. Infiltration of the thoracic wall follows, with an overlying abscess at the dependent part of the pleural cavity. The mediastinal type more readily invades the thoracic wall, with consequent early abscess formation. Pleural effusion and empyema are equally early features. The bodies of the thoracic vertebrae are often involved, as in Case 2.

Bacteriology.-A. israeli has long been considered the sole causative organism. In 1878 Israël found coccoid formation in the "sulphur granules" of actinomycotic pus. $\mathrm{He}$ thought these were a developmental phase of the fungus. Wright (1905) described the frequent presence of other bacteria often intimately associated with the substance of granules. He believed that sometimes the accompanying bacteria play an important part in the extension of the disease. Klinger (1912) found a Gram-negative aerobic coccobacillus in 5 out of 12 cases. He called this organism Bacillus actinomycetem comitans, and subsequently it has been noticed in association with actinomycosis (Colebrook, 1920 ; Bayne-Jonés, 1925 ; Bates, 1933 ; Goldsworthy, 1938 ; Clemens, 1940). Holm (1950) found "other organisms," including this actinobacillus, in the pus from 650 closed actinomycotic lesions. The type of organism varied with the site affected, but in thoracic lesions the actinobacillus was usually present. Also the actinobacillus has been found only in cases of actinomycosis. Colebrook (1920) discussed the significance of this organism, but was unable to find a satisfactory explanation. Holm (1950) believes that actinomycosis is a multiple infection arising from a synergism between $A$. israeli and associated organisms. In this respect it would resemble other infections where there is an association between anaerobic and aerobic organisms-for example, gasgangrene (clostridia and coliforms). We feel that the actinobacillus is significant and plays an important part in the establishment and continuation of the actinomycotic infection.

Mode of Entry.-In 1920 Colebrook stated that the established idea that the ray fungus is conveyed to man from vegetable sources-for example, grasses, soil, etc.-should be viewed with considerable scepticism. It is now generally accepted that $A$. israeli is not the same species as $A$. bovis of cattle actinomycosis nor the species, usually aerobic, found in grasses and soils (Wright, 1905; Cope, 1938 ; Rosebury, 1944 ; Weed and Baggenstoss, 1949). A. israeli is often found in carious teeth and infected tonsils of normal people (Naeslund, 1925 ; Davis, 1941 ; Slack, 1942). It probably requires trauma or locally reduced resistance, with an associated synergic aerobe, to establish a lesion. Pulmonary infection may originate by: inhalation, penetration of the oesophagus, extension from the cervical region via the mediastinum, or retroperitoneal extension from below the diaphragm.

Osteitis. - Infection of bone usually occurs either primarily, as in the jaw, or by extension from neighbouring tissue. Infection by the blood stream is rare, but may cause a lesion in the long bones. In vertebrae involved by local extension of the disease there is, radiologically, irregular rarefaction of the body and surrounding osteosclerosis; angular collapse is rare, and the intervertebral disk is not attacked (Lubert, 1944 ; Shanks and Kerley, 1950). In the ribs a similar process occurs, the earliest change being a periostitis as in Case 1. This gives an interesting and, we feel, characteristic radiological appearance of considerable diagnostic importance.

\section{Treatment}

The sensitivity of $A$. israeli varies in vitro (Strauss et al., 1951), but it has been found that the majority of strains are sensitive to less than $\mathbf{0 . 5}$ microgram (approximately 0.75 unit) per ml. of penicillin (Garrod, 1952). The Actinobacillus actinomycetem comitans is normally insensitive to penicillin but sensitive to streptomycin, as in our Case 2. For this reason both antibiotics were given. In spite of 6 mega units of penicillin daily for seven weeks and two courses of streptomycin, $1 \mathrm{~g}$. daily for four days, new lesions appeared in both lungs one month after cessation of therapy. The failure of some cases to respond to adequate penicillin therapy may be due to insufficient antibiotic reaching the lesion, as a result of avascularity and fibrosis. This may well occur in actinomycotic involvement of bone. Fisher and Harvey (1956) suggest continuation of penicillin treatment for six months, but this would seem necessary only in recurrent or advanced cases. In these, after initial systemic therapy, continuation with oral penicillin would probably be adequate.

\section{Summary}

Two cases of thoracic actinomycosis are reported. The first of these patients presented with a pleural effusion and died seven years later of adrenal failure due to amyloidosis. The second presented with an empyema necessitatis pointing high in the interscapular region.

The diagnostic importance in thoracic actinomycosis of what we feel to be a characteristic type of osteitis of the ribs is emphasized.

The significance of organisms other than Actinomyces israeli, particularly Actinobacillus actinomycetem comitans, in the pathogenesis of the disease is discussed.

In order to deal effectively with both organisms treatment with penicillin and streptomycin is suggested, and in some cases prolonged therapy may be required.

We thank Dr. B. W. Lacey for the bacteriological findings and his helpful criticism of this paper.

\section{REFERENCES}

Barker, C. S. (1954). Canad. med. Ass. J., 71, 332.

Bates, R. (1933). Lancet, 1, 571.

Bayne-Jones, S. (1925). J. Bact., 10, 569.

Clemens, H. H. (1940). J. Pediat., 16, 487.

Colebrook, L. (1920). Brtt. J. exp. Path., 1, 197.

Cope, Z. (1938). Actinomycosis. Oxford Univ. Press, London.

Davis, M. I. J. (1941). Amer. J. Surg., 52, 447.

Fisher, A. M.; and Harvey, J. C. (1956). Postgrad. Med., 19, 32

Garrod, L. P. (1952). Brit. med. J., 1, 1263.

Goldsworthy, N. E. (1938). J. Path. Bact., 46, 207.

Holm, P. (1950). Acta path. microbiol. Scand., 27, 736.

Israek, J. (1878). Virchows Arch. path. Anat., 74, 15.

Klinger, R. (1912). Zbl. Bakt., I. Abt. Orig.; 62, 191

Lubert, M. (1944). Amer. J. Roentgenol., 51, 669.

Naslund, C. (1925). Acta path. microbiol. Scand, 2, 110.

Rosebury T. (1944).

Shanks, S., and Keriey, P. (1950). Textbook of $X$-ray Diagnosis, 2nd ed., 4. 299. Lewis, London

Strauss $R$. (1951). Amer. Rev. Strauss, R. E., Kligman.

Weed, L. A., and Baggenstoss, A. H. (1949). Amer. J. clin. Path., 19, 201. Wright, J. H. (1905). J. med. Res., 13, 349. 